\title{
Using Tactile Sensing to Improve Performance when Grasping Moving Objects
}

\author{
Patrick Lynch ${ }^{1}$ and Conor McGinn ${ }^{1}$
}

\begin{abstract}
To operate in unstructured settings, robots require control strategies that effectively respond to temporal changes in their environment. In the domain of robotic manipulation, most state-of-the-art approaches assume the objects to be grasped are static. Although some research has investigated the challenges of grasping moving objects, these studies have tended to use grasping plans which require a real-time estimate of a gripper-object interception point, which can be difficult to precisely obtain. The goal of this research was to investigate the performance benefits of using a controller with a reactive component for grasping tasks involving moving objects. We hypothesised that using a reactive control strategy, based on tactile sensing in the fingertips, would lead to improved performance and robustness across a wider range of grasping conditions. To test this hypothesis, the ability of a two-finger gripper to grasp a moving ball under a range of dynamic conditions was tested in simulation using the Gazebo robotic simulator and ODE physics engine. Tests revealed that for controllers where the grasp was not informed by tactile sensing, grasping success dropped significantly with increased misalignment between the centre point of the gripper and the point of interception with the moving object. In contrast, the performance of a reactive grasping strategy based on tactile sensing was found to be more robust across these conditions. These findings indicate that tactile sensing could play an important role in enabling robots to interact more effectively with dynamic objects.
\end{abstract}

\section{INTRODUCTION}

Despite increasing demand, the use of robotic manipulation outside controlled environments (factories, warehouses, etc) remains low. In these settings, factors in the environment can be tailored to reduce the complexity of the grasping challenge (i.e. use of environmental sensors, consistent lighting, clutter-free workspace). While a lot of recent research has focused on improving grasp robustness in tasks involving unfamiliar objects [1], changing lighting [2], and in settings with occlusions [3], [4], relatively little has addressed fundamental aspects of grasping moving objects. The inability for robot manipulators to grasp objects in motion limits the applicability of the technology in both traditional industrial settings, such as on assembly lines where the emergence of flexible manufacturing has driven major improvements in productivity, as well as in nontraditional robotic settings, such as homes, hospitals, and logistics where new applications for mobile manipulators are emerging.

Current state-of-the-art grasping approaches for grasping moving objects have broadly followed the same approach, whereby the robot moves its end-effector to a gripper-object

\footnotetext{
*This work was supported the Irish Research Council

${ }^{1}$ School of Engineering, Mathematics and Science, Trinity College Dublin, Ireland lynchp13etcd.ie
}

interception point, estimated by tracking the objects trajectory [5]-[9]. There are several common problems associated with this approach, owing mainly to the dependence of these systems on computer vision. Vision sensing is computationally expensive and gaining the required accuracy at the point of interception often necessitates the use of distributed motion tracking hardware which is costly and rarely practical in everyday settings. Vision sensing is also subject to issues such as occlusion and poor lighting conditions which can further impact their accuracy. Finally, a robotic manipulator is typically a long, open, kinematic chain, making it difficult to achieve high positional accuracy at its end-effector. Each of these issues contribute to an error in the interception of the object by the gripper.

The aim of this research was to explore if performance could be improved when grasping moving objects through incorporation of reactive grasping behaviour, driven by tactile sensing in the gripper's fingertips. It was hypothesised that this approach would enable the gripper to better adapt to errors at the gripper-object interception point, leading to improved overall grasp performance. To test this hypothesis, a series of grasping tests were performed on a virtual gripper in simulation.

The remainder of this paper is structured as follows. Section II details relevant, existing research in the areas of grasping moving objects and visuotactile systems. Section III describes the simulated gripper and environment used, the grasping strategies which are compared and outlines the experimental procedure. Section IV presents the results obtained from testing. Section V examines the presented results, discusses the findings and outlines future work. Finally, section VI draws conclusions from the research presented.

\section{PRIOR WORK}

Previous research connected with the problem of grasping moving objects has explored catching dropped objects [10], catching a flying object [8], [9], and the ability to grasp irregularly shaped moving objects [5]. A number of other relevant studies have centered on robot participation in games and sport, where the robot was required to autonomously interact with a dynamic object. For example, robots have been trained to play table tennis [7], catch [6], [11] and kendama [12]. In each of these studies, the performance of the robot was critically dependant on the ability of its vision system to accurately track the target moving object and estimate an appropriate interception position and time. 
The combination of vision and tactile sensing has been shown to be effective in grasping applications involving static objects. The fusion of these modalities has been used to monitor the relationship between gripper and grasped object during a drilling operation [13] and to ensure stability when grasping static objects [14]. There has been an explosion in recent years of research aiming to use machine learning techniques to process a combination of vision and tactile sensor data to complete a range of goals, including assessing grasp stability [15], inferring information about the objects surface properties [16], informing and improving a reattempted grasp of a static object [17], and to improve a robot's ability to identify and recognise unknown objects [18], [19].

Visuo-tactile sensing systems have also previously been used to aid in the grasping of moving objects [20]-[22]. One example is manipulation of an object on a conveyor belt [20], [21], however this application is able to leverage some of the advantages of a structured environment. Tracking the object with the gripper simplifies the grasp to a quasistatic problem. More recently, researchers used a visuotactile system to grasp falling objects, aiming to minimise the forces, and therefore deformation, applied to a soft object during grasping [22]. Their grasp adaption strategy was optimized for minimising deformation and did not examine how to adapt the grasping motion in the face of uncertainty in the interception position and time.

The reliance on vision sensing when tackling the problem of grasping moving objects is pervasive throughout the prior art, while the potential contribution of other sensing modalities remains a relatively poorly understood topic. There is a need for fundamental research to help better understand how factors, such as tactile sensing, could enhance the performance of robots in these situations. This research addresses this by examining how tactile feedback can effect the grasp's robustness to errors in the interception of a moving object by a robot.

\section{Methods}

The experiment involved an under-actuated, two finger, pincer gripper grasping a ball moving on a horizontal plane. Each finger possessed 3 tactile sensors, 3 degrees of freedom (Fig. 1a) and was controlled by a single actuator. All tests were performed in simulation. In recent years, simulation has emerged as a popular means of initially testing a research hypothesis in robotics manipulation research, since it is faster and less resource intensive than real-world testing with a physical robot [7], [11], [23]. The simulation environment developed in this research was informed by a number of recent studies involving the simulation of robotic grasping, including [24] and [25].

\section{A. Simulation Environment}

A virtual model of the robotic gripper (Fig. 1b) and a ball was created using the Gazebo robotic simulator (version 9.12.0). The simulation adopted the Open Dynamics Engine (ODE) physics engine and the control interface was implemented using the Robotic Operating System (ROS).

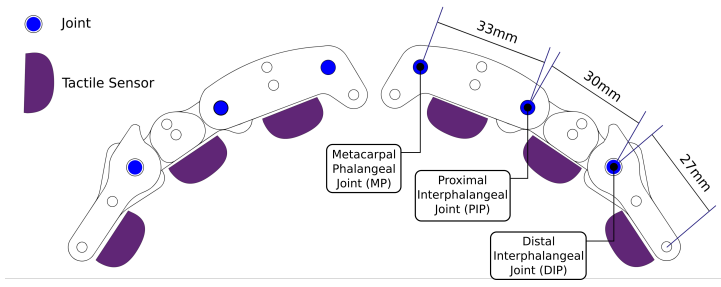

(a)

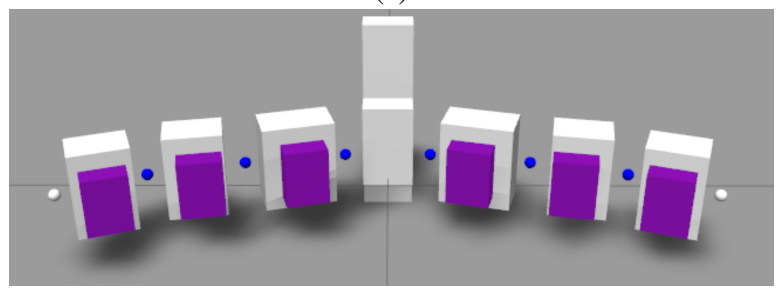

(b)

Fig. 1: Design of gripper: (a) Drawing, with dimensions and legend (b) Screenshot from simulation

The dimensions of the gripper, shown in Fig. 1b, are based on the dimensions of a typical male human hand while the ball is based on a standard billiards ball, with a diameter of $57 \mathrm{~mm}$. Since the ratio of gripper size to ball size will impact performance, the chosen dimension make the results relevant for a humanoid gripper grasping a sphere of similar size to a sports ball which a robot could expect to encounter, i.e. a billiards ball is slightly larger than a golf ball or squash ball but slightly smaller than a baseball, or tennis ball.

A Gazebo plugin was developed to simulate the behaviour of the under-actuated gripper. Each joint was controlled using a velocity PI controller and the torque at each joint was monitored. Initially all joint velocities were set to 0 . When grasping, the plugin provided a step increase in the target velocity of the controller for the first joint. For subsequent joints, only when the torque of the parent joint exceeded an empirically determined threshold, corresponding to when it meets an obstacle in simulation, would the next joint start to move. This behaviour can be seen in Fig. 2b. This is a direct parallel of how the tendons leverage decreasing mechanical advantage for subsequent joints in hardware and results in a sequential closing motion, and inherently adaptive grasp, characteristic of this type of under-actuated gripper.

Tactile sensors were implemented using the built-in gazebo_ros_bumber plugin, which monitors all contacts with each sensor and publishes them to a ROS topic where they can be used by the grasping strategy to inform the grasping motion. Each simulated tactile sensor was modeled using multiple discrete Gazebo links, resulting in multiple contact points per sensor. Tuned dynamic stiffness $\left(k_{p}\right)$ and dynamic damping $\left(k_{d}\right)$ collision parameters, approximated the deformable nature of silicon-based tactile sensors. Furthermore, a non-zero patch and surface radius are used for more accurate simulated frictional behaviour between each sensor and the object. An aspect of the reactive motion, driven by tactile sensors, was the lateral movement of the gripper, 


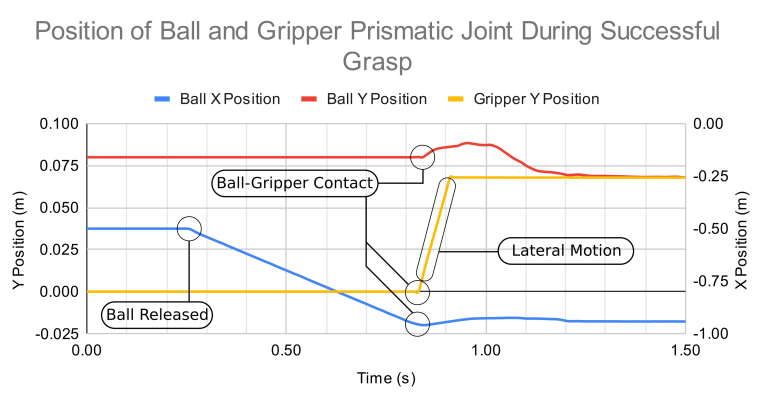

(a)

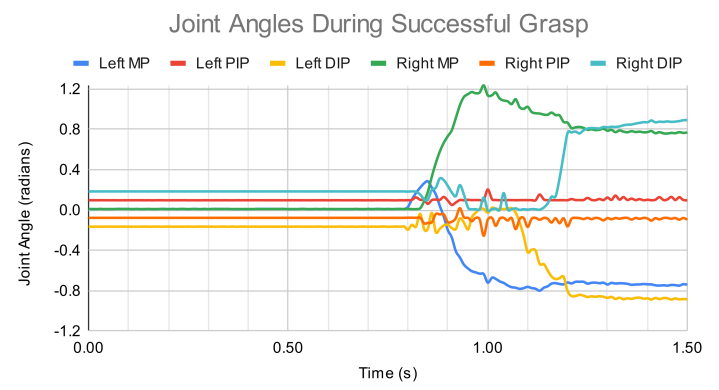

(b)

Fig. 2: Graphs showing (a) ball and gripper position and (b) joint angles, during a successful grasp

illustrated in Fig. 2a. The lateral speed was dependant on tactile sensor feedback and speeds of up to $0.85 \mathrm{~m} / \mathrm{s}$ were used. This was achieved using a gazebo prismatic joint and a position controller which interfaces with ROS through a ROS service call.

\section{B. Experimental Procedure}

The experiment investigated the effectiveness of two distinct control strategies at grasping a moving object. The first strategy involved initiating the grasp a set time before collision, requiring an estimate of the time at which the object would come into contact with the gripper. This provided a proxy to current state-of-the-art approaches that depend exclusively on an estimated interception point to trigger the grasp. A series of experiments were performed to determine the best time to initiate the grasp using this strategy. This involved examining grasp success rates as a function of different ball speeds, ball-gripper contact points and grasp initiation times. The optimal time to initiate the grasp was taken as the point that had the highest overall mean grasp success rate (approximately $0.17 \mathrm{~s}$ before the collision), the results from these simulations is given in Fig. 3. Two other control conditions were taken at $0.30 \mathrm{~s}$ and $0.04 \mathrm{~s}$ before contact (these are equidistant, either side of the optimum) and were also analysed in the experiment. This examines how errors in the estimated optimum time to intercept can effect performance.

The second strategy involved a reactive control component that used feedback from tactile sensing to inform the grasping motion. This was achieved through the implementation of three basic heuristics:

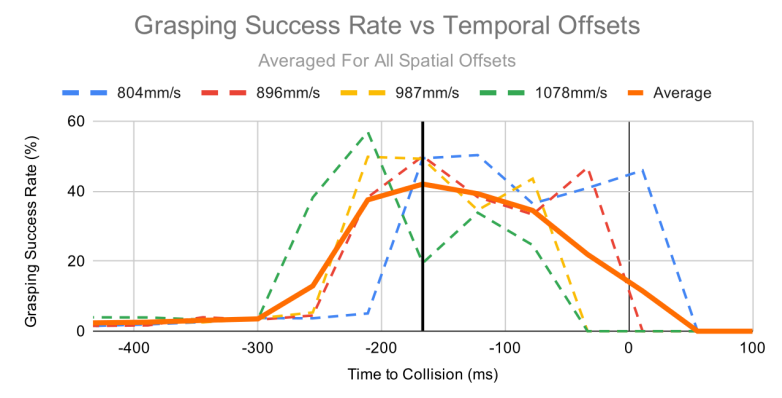

Fig. 3: Initial tests were conducted to determine the optimal grasp initiation time for use in subsequent tests. The optimum time to initiate the grasp was found by determining the grasp initiation time with the highest mean grasp success-rate across different spatial offsets and ball speeds. The optimum time to initiate the grasp was found to be at approximately $0.17 \mathrm{~s}$ before contact as indicated by the vertical black line.

1) The grasp was triggered when the object first made contact with any of the tactile sensors in the gripper.

2) Upon detection of contact with an object, the gripper moved laterally such to reduce the spatial offset and centre the object in the gripper.

3) The closing motion of the finger that first comes into contact with the object is delayed relative to the other finger.

For each test, the ball was spawned at the desired location and programmed to move at the specified speed for the test. The ball moved toward the gripper and the gripper attempted to grasp it. After the attempt, the gripper lifted off the ground plane still attempting to hold the object. A grasp was deemed successful if the gripper could successfully lift and support the full weight of the ball after the grasp.

\section{RESUlTS}

A series of experiments were conducted to investigate the performance of both grasping strategies. Testing was conducted at three different ball speeds, evenly spaced between $0.8 \mathrm{~m} / \mathrm{s}$ and $1 \mathrm{~m} / \mathrm{s}$. Thirteen different spacial offsets were tested, equally spaced between $-69 \mathrm{~mm}$ and $69 \mathrm{~mm}$. These conditions are illustrated in Fig. 4.

Four controllers were implemented in the experiment. The first three controllers used planners that initiated the grasp based on the predicted point of contact between the ball and gripper. These involved initiating the grasp at $0.04 \mathrm{~s}$, $0.17 \mathrm{~s}$ and $0.30 \mathrm{~s}$ from the point of contact respectively. The fourth control condition was the reactive approach, which responded only once the ball had been detected by one of the gripper's tactile sensors. For each test condition, a minimum of 100 tests were conducted and the results at each ball speed are summarised in Fig. 5a - Fig. 5c.

Statistical tests, undertaken using a chi-square test of independence were used to compare the success of the reactive grasping strategy with each of the three control conditions. These results are presented in Table I. Due to the symmetry of the gripper, only results from positive offsets (i.e. offsets 


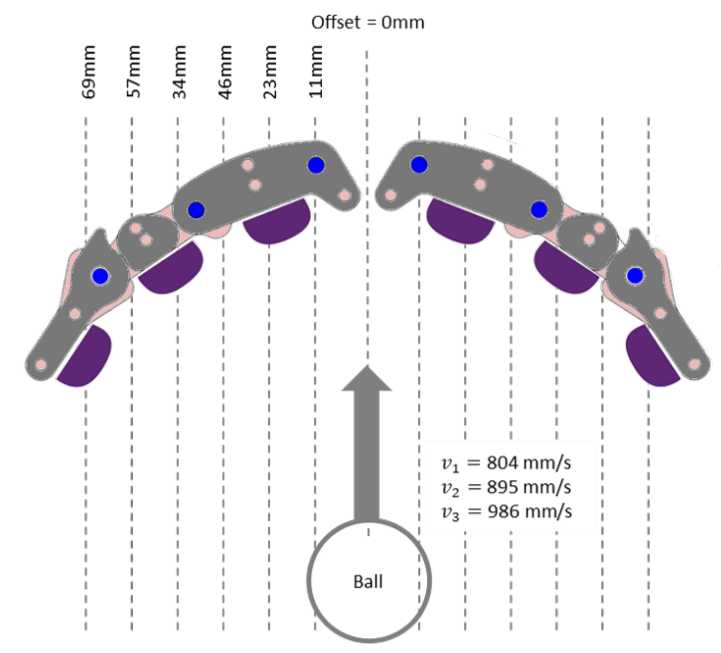

Fig. 4: Tests were performed at 3 ball speeds and 13 spacial offsets as indicated in this graph.

in the range $0-69 \mathrm{~mm}$ ) are presented. It is observed from Table I that the reactive controller outperforms each of the predictive controllers at significant levels for many of the test conditions.

\section{DISCUSSION}

The performance of the control condition (i.e. the approach which relied exclusively on an estimated interception) performed well at low offsets but the grasping success rate dropped as the offset increased. This observation reinforces what was forecast, namely high grasp performance of dynamic objects is only possible using this approach when an accurate estimate of an appropriate interception can be provided. We observed that the performance of this strategy is dependent on the magnitude of the errors (i.e. the distance between the midpoint of the ball and the centre of the gripper) at the point of interception, which would be hard to avoid in many real-world scenarios. This effect is amplified at higher object speeds, where sensitivity to errors was larger and grasp performance observed to be worse, even at small induced errors. In contrast, the reactive grasping strategy demonstrates a robustness to these induced errors, showing the ability to grasp at higher induced spatial offsets, while matching the performance of the estimate-only strategy at lower offsets. This suggests that the techniques implemented as part of the reactive grasping strategy, i.e. the reactive lateral movement and finger coordination, were able to mitigate the effects of an error in the interception, and achieve a successful grasp which would otherwise have been impossible. These techniques are only possible due to the low bandwidth, low latency, and local nature of the implemented tactile sensing and reactive grasping strategy. This reduces the accuracy demands on the estimated interception and is a step toward making such a system effective in an unstructured environment.

The range of spatial offsets which were tested represents the range at which a successful grasp is possible with

\begin{tabular}{|c|c|c|c|c|c|c|c|c|}
\hline & & & & & & & & \\
\hline Offset & Speed & Delay & $\mathrm{n}$ & Success & $\mathrm{n}$ & Success & $\chi^{2}$ & P-val \\
\hline $0 \mathrm{~mm}$ & 0.8044 & $-133 \mathrm{~ms}$ & 107 & $92.52 \%$ & 138 & $93.48 \%$ & 0.001465 & 0.969 \\
\hline $0 \mathrm{~mm}$ & 0.8044 & $\underline{0}$ & 102 & $96.08 \%$ & 138 & $93.48 \%$ & 0.34965 & 0.554 \\
\hline $0 \mathrm{~mm}$ & 0.8044 & $133 \mathrm{~ms}$ & 103 & $9.71 \%$ & 138 & $93.48 \%$ & 166.1328 & $<0.001^{* *}$ \\
\hline $0 \mathrm{~mm}$ & 0.8955 & $-133 m s$ & 106 & $85.85 \%$ & 137 & $89.78 \%$ & 0.544788 & 0.460 \\
\hline $0 \mathrm{~mm}$ & 0.8955 & 0 & 101 & $93.07 \%$ & 137 & $89.78 \%$ & 0.426123 & 0.514 \\
\hline $0 \mathrm{~mm}$ & 0.8955 & $133 \mathrm{~ms}$ & 101 & $5.94 \%$ & 137 & $89.78 \%$ & 161.2701 & $<0.001^{* *}$ \\
\hline $0 \mathrm{~mm}$ & 0.9866 & $-133 \mathrm{~ms}$ & 105 & $0.00 \%$ & 138 & $97.10 \%$ & 223.3889 & $<0.001^{* *}$ \\
\hline $0 \mathrm{~mm}$ & 0.9866 & 0 & 100 & $94.00 \%$ & 138 & $97.10 \%$ & 0.722237 & 0.395 \\
\hline $0 \mathrm{~mm}$ & 0.9866 & $133 \mathrm{~ms}$ & 102 & $8.82 \%$ & 138 & $97.10 \%$ & 186.1479 & $<0.001^{* *}$ \\
\hline $11 \mathrm{~mm}$ & 0.8044 & $-133 \mathrm{~ms}$ & 109 & $93.58 \%$ & 144 & $85.42 \%$ & 3.410192 & 0.065 \\
\hline $11 \mathrm{~mm}$ & 0.8044 & 0 & 102 & $97.06 \%$ & 144 & $85.42 \%$ & 7.917026 & $<0.001^{* *}$ \\
\hline $11 \mathrm{~mm}$ & 0.8044 & $133 \mathrm{~ms}$ & 103 & $8.74 \%$ & 144 & $85.42 \%$ & 138.8327 & $<0.001^{* *}$ \\
\hline $11 \mathrm{~mm}$ & 0.8955 & $-133 \mathrm{~ms}$ & 108 & $88.89 \%$ & 141 & $84.40 \%$ & 0.700228 & 0.403 \\
\hline $11 \mathrm{~mm}$ & 0.8955 & 0 & 103 & $98.06 \%$ & 141 & $84.40 \%$ & 11.03212 & $<0.001^{* *}$ \\
\hline $11 \mathrm{~mm}$ & 0.8955 & $133 \mathrm{~ms}$ & 104 & $6.73 \%$ & 141 & $84.40 \%$ & 141.4406 & $<0.001^{* *}$ \\
\hline $11 \mathrm{~mm}$ & 0.9866 & $-133 \mathrm{~ms}$ & 107 & $0.00 \%$ & 148 & $73.65 \%$ & 134.6444 & $<0.001^{* *}$ \\
\hline $11 \mathrm{~mm}$ & 0.9866 & & 101 & $93.07 \%$ & 148 & $73.65 \%$ & 13.77156 & $<0.001^{* *}$ \\
\hline $11 \mathrm{~mm}$ & 0.9866 & $133 \mathrm{~ms}$ & 107 & $9.35 \%$ & 148 & $73.65 \%$ & 100.6042 & $<0.001^{* *}$ \\
\hline $23 \mathrm{~mm}$ & 0.8044 & $-133 \mathrm{~ms}$ & 105 & $81.90 \%$ & 139 & $97.84 \%$ & 16.62774 & $<0.001^{* *}$ \\
\hline $23 \mathrm{~mm}$ & 0.8044 & 0 & 104 & $94.23 \%$ & 139 & $97.84 \%$ & 1.28028 & 0.258 \\
\hline $23 \mathrm{~mm}$ & 0.8044 & $133 \mathrm{~ms}$ & 103 & $80 \%$ & 139 & $97.84 \%$ & 199. & $<0.001^{* *}$ \\
\hline $23 \mathrm{~mm}$ & 0.8955 & $-133 \mathrm{~ms}$ & 106 & $88.68 \%$ & 136 & $94.85 \%$ & 2.34296 & 0.126 \\
\hline $23 \mathrm{~mm}$ & 0.8955 & 0 & 102 & $92.16 \%$ & 136 & $94.85 \%$ & 0.33352 & 0.564 \\
\hline $23 \mathrm{~mm}$ & 0.8955 & $133 \mathrm{~ms}$ & 103 & $6.80 \%$ & 136 & $94.85 \%$ & 181.7474 & $<0.001^{* *}$ \\
\hline $23 \mathrm{~mm}$ & 0.9866 & $-133 \mathrm{~ms}$ & 107 & $0.00 \%$ & 138 & $93.48 \%$ & 207.5198 & $<0.001^{* *}$ \\
\hline $23 \mathrm{~mm}$ & 0.9866 & & 105 & $96.19 \%$ & 138 & $93.48 \%$ & 0.413434 & 0.520 \\
\hline $23 \mathrm{~mm}$ & 0.9866 & $133 \mathrm{~ms}$ & 100 & $7.00 \%$ & 138 & $93.48 \%$ & 173.5506 & $<0.001^{* *}$ \\
\hline $34 \mathrm{~mm}$ & 0.8044 & $-133 \mathrm{~ms}$ & 110 & $21.82 \%$ & 142 & $68.31 \%$ & 51.82896 & $<0.001^{* *}$ \\
\hline $34 \mathrm{~mm}$ & 0.8044 & 0 & 101 & $68.32 \%$ & 142 & $68.31 \%$ & 0 & 1.000 \\
\hline $34 \mathrm{~mm}$ & 0.8044 & $133 \mathrm{~ms}$ & 103 & $88 \%$ & 142 & $68.31 \%$ & 99.62521 & $<0.001^{* *}$ \\
\hline $34 \mathrm{~mm}$ & 0.8955 & $-133 \mathrm{~ms}$ & 110 & $87.27 \%$ & 141 & $92.91 \%$ & 1.664211 & 0.197 \\
\hline $34 \mathrm{~mm}$ & 0.8955 & 0 & 106 & $90.57 \%$ & 141 & $92.91 \%$ & 0.186747 & 0.666 \\
\hline $34 \mathrm{~mm}$ & 0.8955 & $133 \mathrm{~ms}$ & 104 & $2.88 \%$ & 141 & $92.91 \%$ & 192.1329 & $<0.001^{* *}$ \\
\hline $34 \mathrm{~mm}$ & 0.9866 & $-133 \mathrm{~ms}$ & 109 & $0.00 \%$ & 139 & $93.53 \%$ & 210.5185 & $<0.001^{* *}$ \\
\hline $34 \mathrm{~mm}$ & 0.9866 & & 104 & $91.35 \%$ & 139 & $93.53 \%$ & 0.155405 & 0.693 \\
\hline $34 \mathrm{~mm}$ & 0.9866 & $133 \mathrm{~ms}$ & 106 & $83 \%$ & 139 & $93.53 \%$ & 195.6972 & $<0.001^{* *}$ \\
\hline $46 \mathrm{~mm}$ & 0.8044 & $-133 \mathrm{~ms}$ & 107 & $0.00 \%$ & 141 & $88.65 \%$ & 187.7288 & $<0.001^{* *}$ \\
\hline $46 \mathrm{~mm}$ & 0.8044 & 0 & 103 & $1.94 \%$ & 141 & $88.65 \%$ & 175.8517 & $<0.001^{* *}$ \\
\hline $46 \mathrm{~mm}$ & 0.8044 & $133 \mathrm{~ms}$ & 103 & $0.00 \%$ & 141 & $88.65 \%$ & 183.6966 & $<0.001^{* *}$ \\
\hline $46 \mathrm{~mm}$ & 0.8955 & $-133 \mathrm{~ms}$ & 107 & $0.00 \%$ & 137 & $64.23 \%$ & 104.733 & $<0.001^{* *}$ \\
\hline $46 \mathrm{~mm}$ & 0.8955 & 0 & 103 & $0.97 \%$ & 137 & $64.23 \%$ & 98.16179 & $<0.001^{* *}$ \\
\hline $46 \mathrm{~mm}$ & 0.8955 & $133 \mathrm{~ms}$ & 103 & $0.00 \%$ & 137 & $64.23 \%$ & 101.7164 & $<0.001^{* *}$ \\
\hline $46 \mathrm{~mm}$ & 0.9866 & $-133 \mathrm{~ms}$ & 107 & $0.00 \%$ & 139 & $74.10 \%$ & 133.3696 & $<0.001^{* *}$ \\
\hline $46 \mathrm{~mm}$ & 0.9866 & 0 & 104 & $1.92 \%$ & 139 & $74.10 \%$ & 123.3723 & $<0.001^{* *}$ \\
\hline $46 \mathrm{~mm}$ & 0.9866 & $133 \mathrm{~ms}$ & 103 & $0.00 \%$ & 139 & $74.10 \%$ & 129.8664 & $<0.001^{* *}$ \\
\hline $57 \mathrm{~mm}$ & 0.8044 & $-133 \mathrm{~ms}$ & 108 & $0.00 \%$ & 140 & $57.86 \%$ & 90.18118 & $<0.001^{* *}$ \\
\hline $57 \mathrm{~mm}$ & 0.8044 & 0 & 104 & $0.00 \%$ & 140 & $57.86 \%$ & 87.48252 & $<0.001^{* *}$ \\
\hline $57 \mathrm{~mm}$ & 0.8044 & $133 \mathrm{~ms}$ & 103 & $0.00 \%$ & 140 & $57.86 \%$ & 86.80467 & $<0.001^{* *}$ \\
\hline $57 \mathrm{~mm}$ & 0.8955 & $-133 \mathrm{~ms}$ & 105 & $0.00 \%$ & 136 & $35.29 \%$ & 44.0892 & $<0.001^{* *}$ \\
\hline $57 \mathrm{~mm}$ & 0.8955 & 0 & 103 & $0.00 \%$ & 136 & $35.29 \%$ & 43.31634 & $<0.001^{* *}$ \\
\hline $57 \mathrm{~mm}$ & 0.8955 & $133 \mathrm{~ms}$ & 102 & $0.00 \%$ & 136 & $35.29 \%$ & 42.92927 & $<0.001^{* *}$ \\
\hline $57 \mathrm{~mm}$ & 0.9866 & $-133 \mathrm{~ms}$ & 106 & $0.00 \%$ & 137 & $5.84 \%$ & 4.697786 & $0.030^{*}$ \\
\hline $57 \mathrm{~mm}$ & 0.9866 & 0 & 103 & $0.00 \%$ & 137 & $5.84 \%$ & 4.541725 & $0.033^{*}$ \\
\hline $57 \mathrm{~mm}$ & 0.9866 & $133 \mathrm{~ms}$ & 101 & $0.00 \%$ & 137 & $5.84 \%$ & 4.437676 & $0.035^{*}$ \\
\hline $69 \mathrm{~mm}$ & 0.8044 & $-133 \mathrm{~ms}$ & 110 & $0.00 \%$ & 166 & $40.36 \%$ & 56.45521 & $<0.001^{* *}$ \\
\hline $69 \mathrm{~mm}$ & 0.8044 & 0 & 105 & $0.00 \%$ & 166 & $40.36 \%$ & 54.15046 & $<0.001^{* *}$ \\
\hline $69 \mathrm{~mm}$ & 0.8044 & $133 \mathrm{~ms}$ & 100 & $0.00 \%$ & 166 & $40.36 \%$ & 51.82982 & $<0.001^{* *}$ \\
\hline $69 \mathrm{~mm}$ & 0.8955 & $-133 \mathrm{~ms}$ & 109 & $0.00 \%$ & 168 & $30.36 \%$ & 38.56063 & $<0.001^{* * *}$ \\
\hline $69 \mathrm{~mm}$ & 0.8955 & 0 & 103 & $0.00 \%$ & 168 & $30.36 \%$ & 36.5549 & $<0.001^{* *}$ \\
\hline $69 \mathrm{~mm}$ & 0.8955 & $133 \mathrm{~ms}$ & 104 & $0.00 \%$ & 168 & $30.36 \%$ & 36.89002 & $<0.001^{* *}$ \\
\hline $69 \mathrm{~mm}$ & 0.9866 & $-133 \mathrm{~ms}$ & 108 & $0.00 \%$ & 138 & $0.00 \%$ & NA & NA \\
\hline $69 \mathrm{~mm}$ & 0.9866 & 0 & 103 & $0.00 \%$ & 138 & $0.00 \%$ & NA & NA \\
\hline $69 \mathrm{~mm}$ & 0.9866 & $133 \mathrm{~ms}$ & 103 & $0.00 \%$ & 138 & $0.00 \%$ & NA & NA \\
\hline
\end{tabular}

TABLE I: Summary of results from simulated grasping experiments. Where 'delay' is the grasp time minus the optimum time, ' $n$ ' is the number of tests conducted, $\chi^{2}$ is the chi-squared value and $P-v a l$ is the probability that there is no different between the control and reactive strategies. A single asterisk indicates a significant difference in performance at a significance level $(\alpha)<0.05$, a double asterisk indicates a significant difference in performance at $\alpha<0.01$ 


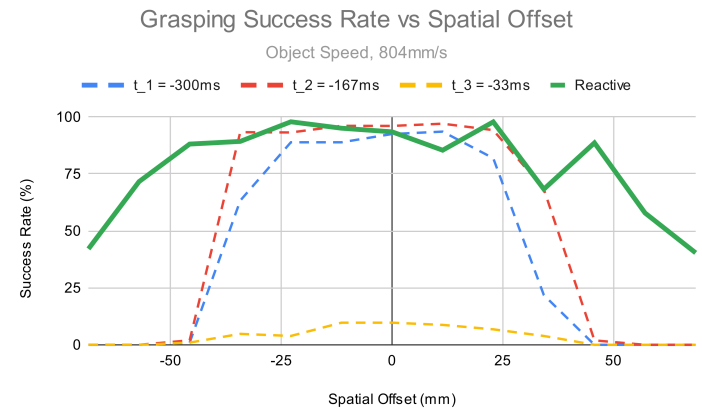

(a)

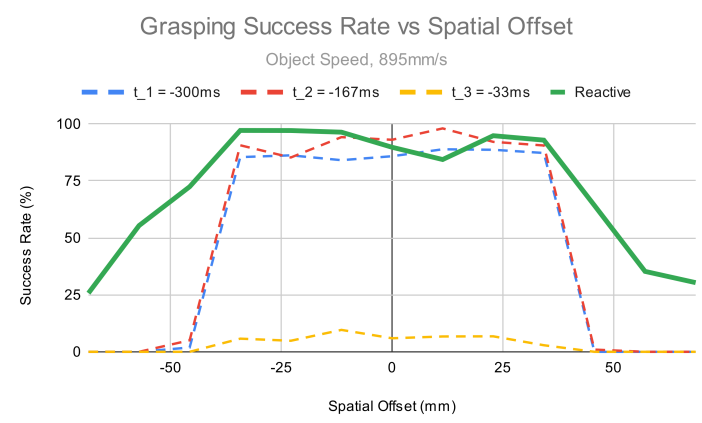

(b)

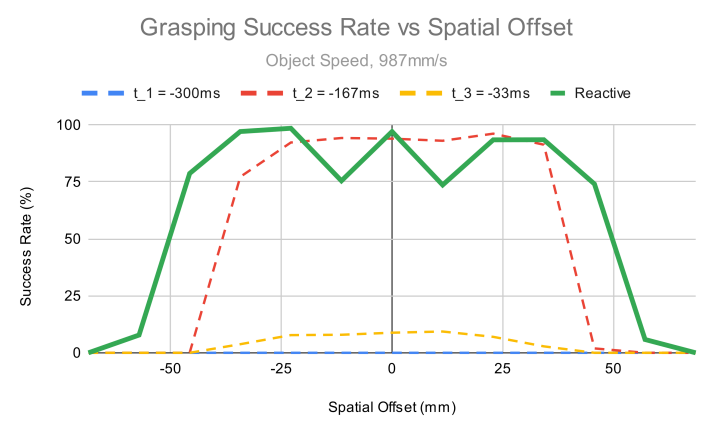

(c)

Fig. 5: Results for different speeds of the moving object: (a) Slow speed, (b) Medium speed, (c) High Speed

this gripper, beyond $69 \mathrm{~mm}$ the grasping success rate drops to zero. To understand the conditions which may lead to interception offsets in the range tested, a theoretical analysis of the trajectory of a flying ball was conducted using a purely gravity-based model, neglecting air resistance. It was found that for a ball thrown at a $45^{\circ}$ angle toward the robot from two meters away, a $2 \%$ error in the measurement of the object velocity, both in magnitude and angle of the velocity vector, when extrapolated will lead to an error in the estimated point of interception of approximately $76 \mathrm{~mm}$. This represents grasping under poor conditions where the robot must extrapolate from sensor data collected when the ball is relatively far from the robot. That said, due to occlusion and poor lighting conditions commonly experienced in unstructured environments it is not an unrealistic scenario. Furthermore, this only accounts for errors in the estimated interception point and not errors in the robot's ability of move the gripper to this position.

The optimum time to initiate the grasp was defined as the time which achieved the highest average grasping success rate, and was determined empirically to be $0.17 \mathrm{~s}$ before interception. However, results suggest that there may be alternative ways in which to define this, depending on the context. Fig. 3 demonstrates that grasping performance deteriorates quicker when the grasp is early compared to when the grasp is late. An alternative way to define the optimum time would be to select a time from which both positive and negative offsets would result in similar performance. Fig. 3 also shows that each speed has a distinct optimum time to initiate the grasp. This suggests that the object speed should be included when calculating the optimum time to initiate the grasp. The data collected shows that this approach could increase the average grasping success rate, from $42 \%$ when the optimum time is defined as an average of all speeds, to $52 \%$ when each tested speed has a distinct optimum time.

In Fig. 5a, it is worth noting some asymmetry in the grasping success rate for the reactive strategy with positive spatial offsets (to the right of optimum) at lower speeds. This feature became apparent during analysis of the results performed, and on reflection is likely due to a slight asymmetry in the alignment of the tactile sensors in simulation. This does not effect the conclusions drawn from this research, and a conservative view of the results was taken when conducting statistical analysis. Positive spatial offsets were considered, rather than the potentially more favourable negative spatial offsets. This does however underlie the importance of sensor position.

In Fig 5c, there is a distinctive drop in performance for low spatial offset, high object speed, test conditions. The grasping motion, as determined by the reactive grasping strategy, was to move the gripper laterally so as to reduce the distance between the center of the gripper and the initial contact point. However, at these low offsets it was found that the rebound resulted in the object crossing the center line of the gripper, resulting in movement of the gripper away from the target object. This suggests that, despite performance improvements at higher offsets, the reactive strategy presented does not necessary react optimally and would achieve yet better results through further refinement. One example of how this might be achieved is by increasing the density of tactile sensing. Higher resolution information about the contact point, would allow the strategy to identify the scenarios in which the object is likely to rebound across the center line of the gripper and trigger a more appropriate response.

An improvement in grasp robustness is shown using a relatively simple tactile sensing implementation. Improvements in both sensor density and sensitivity have the potential to further improve the gripper performance while grasping moving objects and is the subject of future research. There are also several parameters related to grasp uncertainty that remain to be examined, such as variation in the angle at which the gripper intercepts the object and the spin of the object. Further optimization of the time to initiate the grasp, 
in particular the effect of the object speed, is also a topic for future research. The simulation environment presented in this work provides an ideal test-bed for tackling these questions. Current modeling is simplified to a $2 \mathrm{D}$ problem of a ball rolling on a horizontal plane. This is valuable for initial testing and development of the hypothesis, but the benefits of this approach will be best realized when the methodology is applied to 3D grasping. Validation of the results presented here in a real-world experiment is a necessity and is the topic of ongoing research.

\section{Conclusions}

This research hypothesised that using a reactive control strategy, based on tactile sensing in the fingertips, could lead to improved grasp robustness when grasping moving objects. In theory, real-time, tactile feedback could detect errors in the interception of the object by the gripper and enable a grasping motion which reacts appropriately. This results in successful grasps in situations where interception errors would make a successful grasp impossible for a strategy which solely relies on an estimation of the point of interception.

This was tested in simulation, where a two finger gripper attempted to grasp a ball rolling on a horizontal plane. Two different strategies were compared, first a traditional approach, common in prior work on this problem, which relied exclusively on an estimate of a position and time of interception. Second, a reactive strategy which used feedback from tactile sensors to adapt the grasping motion. Results demonstrate that the reactive strategy was able to mitigate the effects of errors at the point of interception in a way which was impossible for the traditional strategy. This showed a clear improvement in the robustness of the strategy to interception errors.

This research represents early stage validation that tactile sensing can play a role in enabling robots to autonomously interact with dynamic objects. To enable the research presented here to have a real impact on enabling robots to interact with dynamic objects, it is essential that these results are replicated in the real world and similar strategies are applied to more complex grasping scenarios.

\section{REFERENCES}

[1] Q. Lei, J. Meijer, and M. Wisse, "A survey of unknown object grasping and our fast grasping algorithm-c shape grasping," in 2017 3rd International Conference on Control, Automation and Robotics (ICCAR), pp. 150-157, 2017.

[2] J. Tremblay, T. To, B. Sundaralingam, Y. Xiang, D. Fox, and S. Birchfield, "Deep object pose estimation for semantic robotic grasping of household objects," 2018.

[3] Y. Yu, Z. Cao, S. Liang, W. Geng, and J. Yu, "A novel vision-based grasping method under occlusion for manipulating robotic system," IEEE Sensors Journal, vol. 20, no. 18, pp. 10996-11006, 2020.

[4] H. Cuevas-Velasquez, N. Li, R. Tylecek, M. Saval-Calvo, and R. B. Fisher, "Hybrid multi-camera visual servoing to moving target," in 2018 IEEE/RSJ International Conference on Intelligent Robots and Systems (IROS), pp. 1132-1137, 2018.

[5] S. Kim, A. Shukla, and A. Billard, "Catching objects in flight," IEEE Transactions on Robotics, vol. 30, no. 5, pp. 1049-1065, 2014.

[6] J. Kober, M. Glisson, and M. Mistry, "Playing catch and juggling with a humanoid robot," IEEE-RAS International Conference on Humanoid Robots, pp. 875-881, 2012.
[7] O. Koç, G. Maeda, and J. Peters, "Online optimal trajectory generation for robot table tennis," Robotics and Autonomous Systems, vol. 105, pp. 121-137, 2018.

[8] B. Bäuml, O. Birbach, T. Wimböck, U. Frese, A. Dietrich, and G. Hirzinger, "Catching flying balls with a mobile humanoid: System overview and design considerations," in 2011 11th IEEE-RAS International Conference on Humanoid Robots, pp. 513-520, 2011.

[9] S. S. M. Salehian, M. Khoramshahi, and A. Billard, "A dynamical system approach for softly catching a flying object: Theory and experiment," IEEE Transactions on Robotics, vol. 32, no. 2, pp. 462471, 2016.

[10] Y. Imai, A. Namiki, K. Hashimoto, and M. Ishikawa, "Dynamic active catching using a high-speed multifingered hand and a high-speed vision system," in IEEE International Conference on Robotics and Automation, 2004. Proceedings. ICRA '04. 2004, vol. 2, pp. 18491854 Vol.2, April 2004.

[11] D. Carneiro, F. Silva, and P. Georgieva, "The role of early anticipations for human-robot ball catching," in 2018 IEEE International Conference on Autonomous Robot Systems and Competitions (ICARSC), pp. 10-16, 2018.

[12] A. Namiki and N. Itoi, "Ball catching in kendama game by estimating grasp conditions based on a high-speed vision system and tactile sensors," IEEE-RAS International Conference on Humanoid Robots, vol. 2015-Febru, pp. 634-639, 2015.

[13] P. Hebert, N. Hudson, J. Ma, T. Howard, T. Fuchs, M. Bajracharya, and J. Burdick, "Combined shape, appearance and silhouette for simultaneous manipulator and object tracking," in 2012 IEEE International Conference on Robotics and Automation, pp. 2405-2412, May 2012.

[14] V. Kumar, T. Hermans, D. Fox, S. Birchfield, and J. Tremblay, "Contextual reinforcement learning of visuo-tactile multi-fingered grasping policies," ArXiv, vol. abs/1911.09233, 2019.

[15] Y. Bekiroglu, A. Damianou, R. Detry, J. A. Stork, D. Kragic, and C. H. Ek, "Probabilistic consolidation of grasp experience," in 2016 IEEE International Conference on Robotics and Automation (ICRA), pp. 193-200, 2016.

[16] Y. Gao, L. A. Hendricks, K. J. Kuchenbecker, and T. Darrell, "Deep learning for tactile understanding from visual and haptic data," IEEE International Conference on Robotics and Automation (ICRA), pp. 536-543, 2016.

[17] E. Kolycheva née Nikandrova and V. Kyrki, "Task-specific grasping of simiiar objects by probabiiistic fusion of vision and tactiie measurements," in 2015 IEEE-RAS 15th International Conference on Humanoid Robots (Humanoids), pp. 704-710, Nov 2015.

[18] M. Bauza, O. Canal, and A. Rodriguez, "Tactile mapping and localization from high-resolution tactile imprints," in 2019 International Conference on Robotics and Automation (ICRA), pp. 3811-3817, 2019.

[19] S. Funabashi, Y. Gang, A. Geier, A. Schmitz, and S. Sugano, "Morphology-Specific Convolutional Neural Networks for Tactile Object Recognition with a Multi-Fingered Hand," International Conference on Robotics and Automation (ICRA), pp. 57-63, 2019.

[20] S. Escaida Navarro, D. Weiss, D. Stogl, D. Milev, and B. Hein, "Tracking and grasping of known and unknown objects from a conveyor belt," in ISR/Robotik 2014; 41st International Symposium on Robotics, pp. 1-8, 2014.

[21] R. C. Luo and C. Liao, "Robotic conveyor tracking with dynamic object fetching for industrial automation," in 2017 IEEE 15th International Conference on Industrial Informatics (INDIN), pp. 369-374, July 2017.

[22] K. Koyama, K. Murakami, T. Senoo, M. Shimojo, and M. Ishikawa, "High-Speed, Small-Deformation Catching of Soft Objects Based on Active Vision and Proximity Sensing," ICRA, vol. 4, no. 2, pp. 578 585, 2019.

[23] K. Kleeberger, R. Bormann, W. Kraus, and M. F. Huber, "A survey on learning-based robotic grasping," Current Robotics Reports, vol. 1, pp. 239-249, Dec 2020.

[24] S. Joshi, S. Kumra, and F. Sahin, "Robotic grasping using deep reinforcement learning," in 2020 IEEE 16th International Conference on Automation Science and Engineering (CASE), pp. 1461-1466, 2020.

[25] A. Mohan, G. Soman, S. S, and J. Cletus, "Design and simulation of 3 fingered underactuated gripper," in 2020 IEEE Recent Advances in Intelligent Computational Systems (RAICS), pp. 86-90, 2020. 